\title{
The effectiveness of current methods of surgical treatment of anterior recurrent instability of the shoulder joint, depending on anatomical and functional changes
}

\author{
N.N. Chirkov ${ }^{1,2}$, N.S. Nikolaev ${ }^{1,2}$, A.V. Alekseeva ${ }^{1}$, V.N. Yakovlev ${ }^{1}$, A.V. Lushin ${ }^{2}$ \\ ${ }^{1}$ Federal Center for Traumatology, Orthopedics and Endoprosthetics, Cheboksary, Russian Federation \\ ${ }^{2}$ Chuvash State University named after I.N. Ulyanov, Cheboksary, Russian Federation
}

\begin{abstract}
Introduction Traumatic anterior dislocation of the shoulder usually results in chronic recurrent shoulder instability, especially in young adults. To reduce the rate of recurrent dislocations, various options of osteoplastic operations have been proposed. The purpose of our study is to determine the significance of anatomical and functional disorders in patients with anterior shoulder joint instability; to evaluate the results of the methods for surgical treatment of patients with anterior recurrent instability of the shoulder joint, depending on the anatomical and functional disorders. Materials and methods The results of diagnostic examination and treatment of 169 patients with recurrent post-traumatic dislocation of the shoulder who were operated on at the Federal State Budgetary Organization "Federal Center for Traumatology, Orthopedics and Arthroplasty" (Cheboksary) were analyzed. The average age of patients was $31.1 \pm 13.1$ years; the average period of postoperative followup was $49.5 \pm 24.0$ months. Clinical data were assessed by angulometry, using the ROWE, ASES, UCLA, VAS scales. The size of bone defects was determined using radiological methods. 99 patients (58.6\%) underwent arthroscopic Bankart repair, 24 patients $(14.2 \%)$ with more than $15 \%$ anterior glenoid bone defect underwent Latarjet operation. Bankart + Remplissage surgery was performed in 39 patients $(23.1 \%)$, Latarjet + Remplissage in 7 (4.1\%) patients. Results Bone defect of the glenoid of more than $15 \%$ of the area was detected in 32 patients. Hill-Sachs grade 3 injury was reported in 24 patients. Most of these patients had a history of more than 20 dislocation episodes. Hyperelasticity of the shoulder joint was found in 34 patients. In the postoperative period, there was a slight improvement in the mean indicators of flexion and abduction in the shoulder joint from $174.2 \pm 15.9^{\circ}$ and $170.6 \pm 17.8^{\circ}$ to $175.6 \pm 9.3^{\circ}$ and $173.0 \pm 12.3^{\circ}$, respectively. The mean external rotation worsened from $61.6 \pm 25.7^{\circ}$ to $55.9 \pm 14.4^{\circ}$. Indicators of the ROWE, ASES, UCLA rating scales improved significantly from $38.5 \pm 8.5$, $76.0 \pm 13.2$ and $20.8 \pm 3.0$ to $89.9 \pm 17.5,94.0 \pm 7.1$ and $32.7 \pm 3.7$ points, respectively. Poor results were observed in 7 patients $(4.1 \%)$, of which three had a relapse. Conclusions Critically significant anatomical and functional disorders in patients with anterior instability of the shoulder joint are the defect of the anterior rim of the articular process of the scapula more than 15 $\%$, the defect of the posterior surface of the humeral head (Hill-Sachs defect) of grade 3, hyperelasticity of the soft tissues of the shoulder joint. The severity of bone defects is directly proportional to the total number of dislocations. The effectiveness of surgical techniques for stabilizing the shoulder joint, depending on the above anatomical and functional disorders, was $95.9 \%$. Keywords: shoulder joint, habitual shoulder dislocation, recurrent instability, Bankart repair, Latarjet operation, remplissage
\end{abstract}

\section{INTRODUCTION}

Traumatic anterior dislocation of the shoulder usually results in chronic recurrent shoulder instability, especially in young adults. The literature reports that the recurrent anterior instability after primary shoulder dislocation occurs in 50 to $96 \%$ of patients who are younger than 20 years of age and in 40 to $74 \%$ of patients in the age from 20 to 40 years. The condition may require surgical treatment in cases of restricted shoulder functions and impairemnt of quality of life [1-3]. Anatomic tear of the anteroinferior labrum of the glenoid along with the joint capsule due to injury (Bankart lesion) creates conditions for recurrent dislocations. Surgical refixation of the cartilaginous labrum to the articular surface is performed with the arthroscopic method and anchor stitching. Thus, the stabilizing effect of the glenoid cavity may be restored (Bankart repair). This type of surgical intervention is anatomical, easy to perform and low invasive and has been considered a "gold standard" for this pathology [4]. However, the study of followups after Bankart shoulder stabilization showed high risks of recurrence in the postoperative period, as the recurrence rates of 4 to $29 \%$ were reported [5-7]. Moreover, patients with shoulder instability associated with an impression fracture of the anterior part of the humeral head (Hill-Sachs lesion) showed high risks of recurrence after classical arthroscopic reconstruction of the capsulo-ligamentous apparatus [8]. To reduce the rate of recurrent dislocations, various options of osteoplastic operations have been proposed. The most popular method is the Latarjet operation that involves the transfer of the osteotomized coracoid process to the defect of the anterior rim of the glenoid. The recurrence following a 5-year period after the Latarjet 
operation was detected in $2.7 \%$ of cases [9]. In large Hill-Sachs defects, the "remplissage" procedure for increasing the stabilization effect was described: performance of anchor tenodesis of the infraspinatus muscle in the area of bone defect of the humeral head $[10,11]$. Wolf E.M. and co-authors report that the recurrence rate after the Bankart repair combined with "remplissage" procedure was much lower and may be less than $5 \%$ [12]. Nevertheless, some authors state that the "remplissage" results in a considerable risk of range of motion restriction in the shoulder [13]. The literature shows that each of the methods to stabilize the shoulder joint has its merits and shortcomings. However, no indications exist that clearly show which stabilization method to apply. In order to differentiate the choice of a surgical method, Balg и Boileau [14] published in 2007 a 10-point system for preoperative evaluation of the shoulder instability severity (ISIS) that considers risk factors that have an impact on a possible recurrence of dislocation after surgical treatment. If the evaluation total is higher than 6 points, the authors do not recommend the Bankart repair due to a high risk of recurrence after its performance.

Miguel Angel Ruiz Ibán and co-authors studied 140 patients (142 joints) at a mean follow-up of 5.3 years after the Bankart repair. All patients had a total ISIS of seven points preoperatively. It was revealed that 117 patients with ISIS points from 0 to 3 had dislocation recurrence of $12.8 \%$; while 25 patients with ISIS points from 4 to 6 had $20 \%$ of recurrence [15].

Joo $\mathrm{Han} \mathrm{Oh}$ and co-authors retrospectively studied the treatment outcomes after the Bankart repair in 120 patients with anterior instability. Among them, 10 patients had a total of seven or more ISIS points (group I), and the remaining 110 patients had 6 or less points (group II). Mean period of control was $23.4 \pm 7.8$ years. Recurrence was observed in 12 patients and 14 more had symptoms of shoulder instability that were also referred to poor outcomes. Among patients of group I, postoperative instability was noted in $30 \%$ of cases while in group II it was $20.9 \%$. Moreover, the authors did not find significant differences in poor results between the groups. The study concluded that this system of evaluation was not reliable in choosing the surgical method [16].

The current theory of instability mechanism called "glenoid track" has been popular. The main idea of the theory is the assessment of the sizes of bipolar bone defects and their impact on the mechanism of dislocation. Thus, the authors recommend making a choice in favor of bone plasty operation in the cases if shoulder bone defect are involved in the dislocation mechanism [17]. However, this theory considers only defects of bone tissue and does not evaluate the soft tissues conditions. Therefore, the problem of studying the anatomic and functional disorders in anterior chronic recurrent shoulder instability to choose among the surgical methods remains unsolved. It is evident that the proper use of current treatment techniques for recurrent shoulder instability would help restore the joint anatomy and the surgeon to obtain good functional results [18].

The purpose of our study was to determine the severity of anatomical and functional disorders in patients with anterior shoulder joint instability; to evaluate the results of the methods for surgical treatment of patients with anterior recurrent instability of the shoulder joint, depending on the anatomical and functional disorders.

\section{MATERIAL AND METHODS}

This retrospective analysis followed the ethical principles of World Medical Association Declaration of Helsinki on Ethical Principles for Medical Research Involving Human Subjects (2013) and rules of clinical practice in the Russian Federation (RF ministry of health order № 266 from 19.06.2003). All patients gave an informed consent on the participation in the study. Evaluation of the treatment results of 169 patients with post-traumatic recurrent shoulder instability was performed retrospectively. They underwent surgical treatment at a trauma department of the Federal Center for Traumatology, Orthopedics and Endoprosthetics (Cheboksary, Russia) from 2012 through 2018. Their mean age was $31.1 \pm 13.1$ years (range, $13-77$ years). There were 137 males and 32 females. The majority were of young working age. Home injuries prevailed that were $86(50.9 \%)$ cases, the rest 62 cases $(36.7 \%)$ were injuries sustained due to sport activities.

Primary dislocation was reduced at a medical care unit in 127 subjects (75.1\%); only 69 (40.8\%) among them continued immobilization of their upper limb for one and a half months. Mean number of recurrences before the intervention was $16.7 \pm 13.0$. Duration of the condition was the period between the first dislocation up to admission to our clinic that was $47.1 \pm 22.3$ months (range, 10-125 months) on average.

Preoperative examination included clinical tests to detect instability (apprehension test, relocation test) and detection of tissue hyperelasticity. Positive anterior hyperelasticity in the shoulder was noted in the cases when external rotation of the shoulder reached the frontal plane ( $90^{\circ}$ and more); the inferior weakness of 
the joint capsule was detected with the Gagey test of hyperabduction. Radiography was taken in standard $\mathrm{AP}$ view and AP projection in maximum internal rotation of the shoulder. MRI (113 patients) and CT (56 cases) was used for accurate detection of bone defects and their severity. We prefer to use MRI in our practice. Despite the fact that bone changes are less clear by MRI study, there is an additional possibility to study soft tissues and less radiation loading.

Sagittal MRI-scans capturing the articular surface of the glenoid were used for determination of the size of the bone defect of the anterior articular process of the scapula (Fig. 1).

A circle is drawn along the contours of the inferior and posterior rims of the glenoid, and the chord $\mathrm{C}$ separates a circle segment that corresponds to the defect area of the anterior rim (Fig. 2).

The defect area of the anterior part of the articular process of the scapula is calculated with an on-line service at (https://planetcalc.ru/1421/) and is the area of the circle segment (Fig. 3).

In order to determine the severity of the impression defect in the proximal humerus (Hill-Sachs lesion), Calandra's classification was used [18]: grade 1 - defect on the articular surface of the humerus, not involving the subchondral bone (Fig. 4 a); grade $2-$ defect involving the subchondral bone (Fig. 4 b); grade 3 - large defect in the subchondral bone (Fig. 4 c).

Based on bone defect severity, a comparative calculation was done to determine the role of bone defect in the mechanism of instability according to the "glenoid track" theory. The calculation was performed according to the method described in the work of Momaya A.M. and Tokish J.M. [19].

Intraoperative arthroscopic examination of the shoulder joint was conducted in all the patients. Under the arthroscopic control, humerus abduction and internal rotation were examined. Also, binding of the Hill-Sachs defect with anterior rim of the articular process of the scapula was detected.

Two arthroscopic techniques were used for surgical treatment of chronic recurrent anterior instability: arthroscopic reconstruction of the capsulo-labrum complex of the shoulder (Bankart repair) and Latarjet bone plasty reconstruction of the shoulder joint under arthroscopic control. Remplissage proceduree was added to reinforce the stabilization effect if indicated.

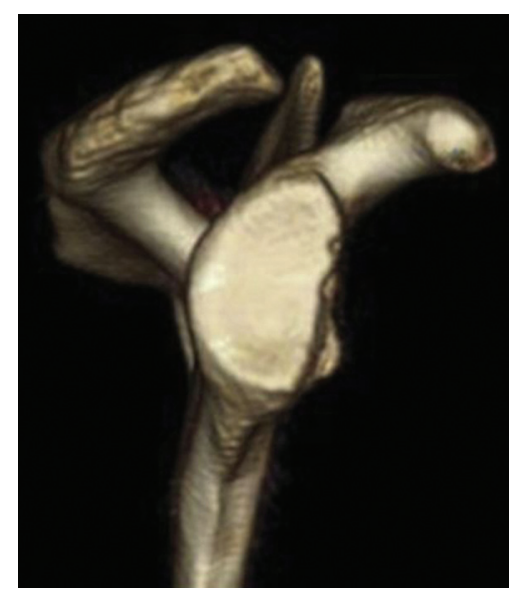

Fig.1 CT 3D-reconstruction with a defect of the anterior rim of the glenoid

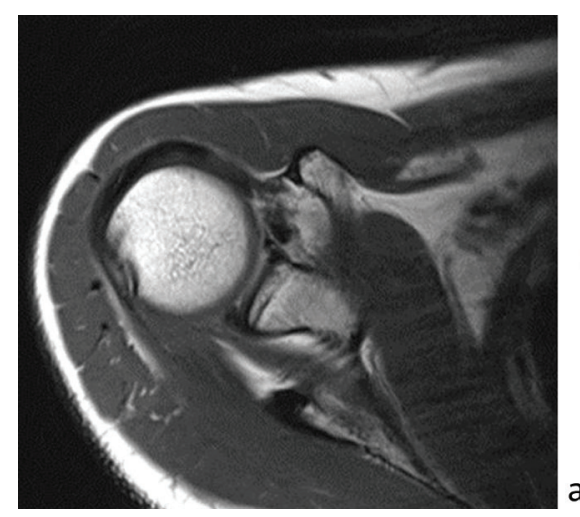

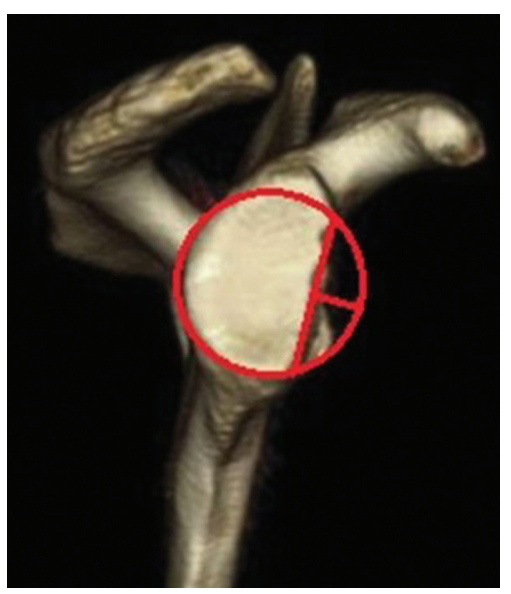

Fig. 2 Determination of the defect area in the anterior rim of the glenoid

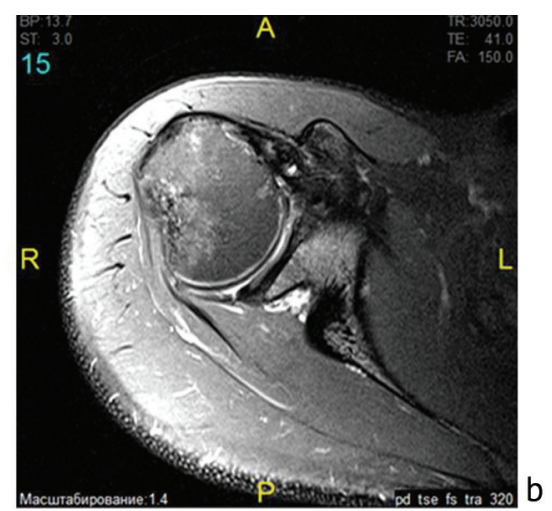

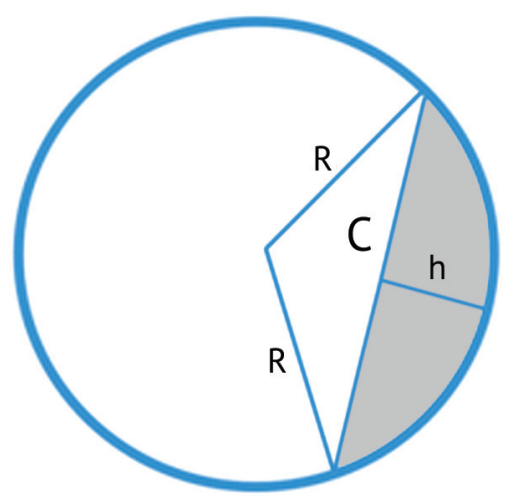

Fig. 3 Geometrical principles of measuring the defect size; $\mathrm{R}$ - radius, $\mathrm{C}$ - chord corresponding to the edge line of bone defects, $\mathrm{h}$ - height of the circle segment. Grey is the defect area to be calculated

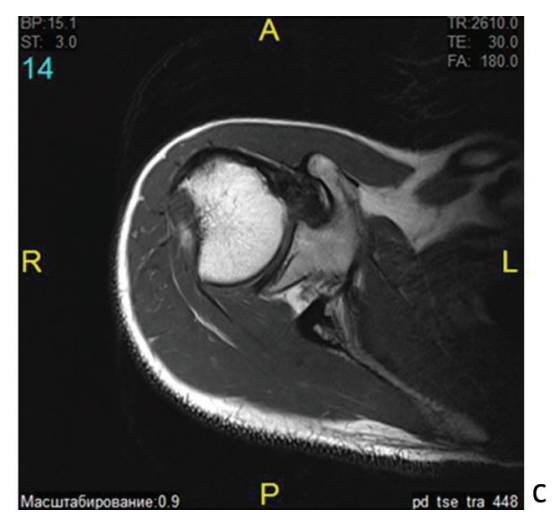

Fig. 4 Defect severity determination with MRI of the shoulder joint: $\boldsymbol{a}$ Hill-Sachs grade $1 ; \boldsymbol{b}$ Hill-Sachs grade 2; $\boldsymbol{c}$ Hill-Sachs grade 3 
ROWE evaluation system was used to assess the results of managing instability that is a ranging point system according the answers to the questions on joint stability, range of motion and functions of the upper limb [20]. The results of ROWE validation system: excellent is a total of 90-100 points; good with $75-89$ point, fair with $51-74$ points and poor with $0-50$ points. Additional evaluation of the shoulder functional state before and after the intervention was done with ASES [21] and UCLA. Pain was assessed with VAS scale.

Work with primary data included filling the "Mediaglog" clinical medical record of each patient; recordings were done at appointed time (before surgery, intraoperatively and in the catamnesis); protocols of study were developed for the time-points studied. Selection of findings into the "Medialog" system was performed according to the set parameters.

Microsoft Excel-2007 was used for statistical processing of the obtained data. Correspondence of the values of the sample to normal distribution in MS Excel was confirmed with diagrams to reflect means (M) and standard deviation (m). Student t-test and Mann-Whitney U-test were used for statistical significance of mean values at $95 \% \mathrm{CI}(\mathrm{p}<0.05)$.

\section{RESULTS}

Apprehension test and relocation test were positive in the majority of cases preoperatively. Apprehension test was positive in 160 patients $(94.7 \%)$ and relocation test in 145 patients $(85.8 \%)$. Hyperelasticity of the shoulder soft tissues was revealed in 34 patients $(20.1 \%)$.

Findings of the mean values of the range of motion and functional results of the preoperative tests are given in Table 1.

Radiographic changes in the shoulder joints in pathological dislocation were detected only in 58 cases from 169 . The maximum severity and number of signs was identified in the subjects with a long history of the disease and high frequency of dislocation. Radiological features of the pathological changes in the shoulder joint of the patients with habitual dislocation of the shoulder are presented in Table 2.

Thus, the majority of patients did not show any bone pathology of the shoulder joint by standard radiographic study.

CT/MRI study revealed that the mean size of the bone defect of the anterior rim of the articular process of the scapula in 169 patients was $7.4 \%$. Defect of more than $15 \%$ of the glenoid area was detected in 32 patients $(18.9 \%)$. The maximal local defect of the posterior surface of the proximal humerus (Hill-Sachs lesion of grade 3) was identified in 24 patients (14.2\%).
The analysis of CT/MRI findings in different disease periods in different patients allowed us to make a conclusion that there is a direct relationship between the number of dislocations in a patient and bone defect severity. In the first months of the disease, bone pathology was not detected or was minimal. Bone changes in the anteroinferior part of the articular process of the scapula and posterolateral parts of the humeral head appeared as the number of dislocations grew (Fig. 5). In the course of time, manifestation of the signs listed above was more evident and there appeared signs of arthritic changes and subluxation of the humeral head.

Thus, the more joint dislocations occur over time, the more pronounced are the bone defects in the shoulder joint. Therefore, it is advisable to stabilize the joint as early as possible, not waiting for the development of a bone defect.

Table 1

Mean values of the range of motion and functional tests before the surgery

\begin{tabular}{|l|c|}
\hline \multicolumn{1}{|c|}{ Parameter } & Value \\
\hline Mean flexion, $^{\circ}$ & $174.2 \pm 15.9$ \\
\hline Mean abduction, $^{\circ}$ & $170.6 \pm 17.8$ \\
\hline Mean external rotation, $^{\circ}$ & $61.6 \pm 25.7$ \\
\hline Hyperelasticity (subjects) $^{\circ}$ & 34 \\
\hline ROWE, points $^{\circ}$ & $38.5 \pm 8.5$ \\
\hline ASES, points & $76.0 \pm 13.2$ \\
\hline UCLA, points & $20.8 \pm 3.0$ \\
\hline
\end{tabular}

Table 2

Frequency of pathological changers in the shoulder according to radiographic study

\begin{tabular}{|l|c|c|}
\hline \multicolumn{1}{|c|}{ Manifestation signs } & $\begin{array}{c}\text { Number } \\
\text { of cases }\end{array}$ & $\begin{array}{c}\% \text { from the total } \\
\text { of cases }\end{array}$ \\
\hline $\begin{array}{l}\text { Discontinuity of the contours or disturbance of the sclerotic line along the antero- } \\
\text { inferior rim of the articular process of the scapula }\end{array}$ & 28 & 16.6 \\
\hline $\begin{array}{l}\text { Deformity of the femoral head in the posterior upper lateral part seen as impression } \\
\text { defect (Hill-Sachs lesion) }\end{array}$ & 42 & 24.8 \\
\hline Signs of deforming arthritis of the scapulohumeral joint in grade 1 & 23 & 13.6 \\
\hline Incongruence of the articular surfaces & 18 & 10.6 \\
\hline
\end{tabular}




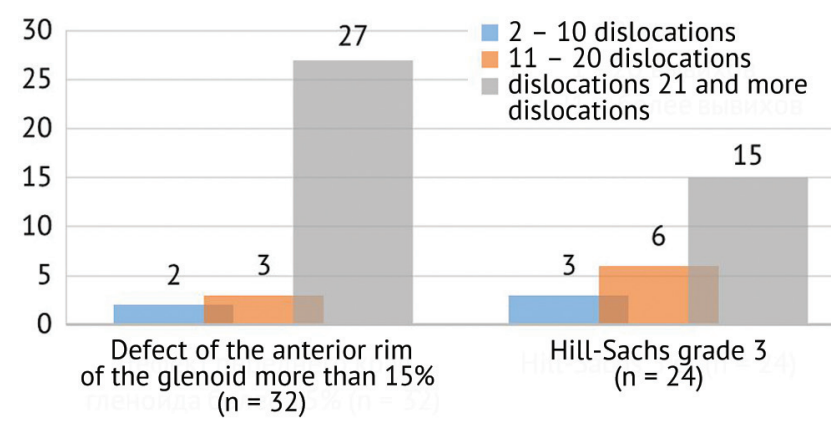

Fig. 5 Relationship of bone defect size in the shoulder joint and numbrt of previous dislocations

We also assessed the relationship of bone defects according to the theory of "glenoid track". Thus, 32 patients $(18.9 \%)$ had bone defects that are significant in the mechanism of instability (glenoid off track), and 137 (81.1\%) had glenoid on track. Comparison of the results of CT/MRI studies showed that the combination of a defect in the anterior rim of the glenoid of more than $15 \%$ with a Hill-Sachs defect of grade 3 caused the "glenoid off track" in all patients. Most of the patients with "glenoid off track" had a defect in the anterior rim of the glenoid of more than $15 \%$ (Table 3 ).

In all patients with anterior instability who underwent an MRI study ( $\mathrm{n}=113$ ), in addition to the above changes, there was a tear of the antero-inferior part of the cartilaginous labrum of the articular process of the scapula with fragment displacement. All patients had complex injuries: tear of the cartilaginous labrum and middle and lower glenohumeral ligaments with overstretching of the anterior joint capsule. In 46 patients, labrum tear was accompanied by synovial cysts. In 11 patients, the cartilaginous labrum was separated from the anterior rim of the glenoid together with a small bone fragment. In four patients, a concomitant pathology was diagnosed with damage to the anterior-superior segment of the articular labrum with a tear of the biceps tendon attachment site.

In all patients with hyperelasticity of the anterior capsule, dislocations in the shoulder joint were observed during diagnostic arthroscopy due to the contact of the Hill-Sachs defect with the anterior rim the glenoid in abduction and external rotation of the shoulder $90^{\circ}$ or more (Fig. 6). It should be noted that this feature was detected in 9 patients with "glenoid on track".

The results of our diagnostic arthroscopy show that hyperelasticity in the joint also requires surgical correction to prevent recurrence. Thus, the findings enable to identify critically significant anatomical and functional disorders affecting stability in the shoulder joint. The algorithm for choosing the surgical technique was based on the assessment of the severity of the changes in the shoulder joint.

When an isolated tear of the anterior cartilaginous labrum was detected, it was re-fixed. In the presence of a bone defect in the anterior rim of the glenoid in a volume of more than $15 \%$, the bone defect was managed with a coracoid graft (Latarjet operation). The presence of a pronounced defect-impression on the posterior surface of the humeral head (HillSachs grade 3) or hyperelasticity of the joint capsule required an additional remplissage procedure, i.e. anchor fixation of regional tissues in the defect. The applied algorithm for choosing a surgical treatment technique in patients with chronic anterior shoulder instability is presented in Table 4.

Number of surgeries performed in the patients with shoulder joint instability, depending on the anatomical and functional changes, is shown in Figure 7.

Table 3

Distribution of patients depending on the severity of bone defects of the shoulder joint and "glenoid track"

\begin{tabular}{|l|c|c|c|c|c|c|}
\hline \multirow{2}{*}{ Glenoid track, $\mathrm{n}=169$} & \multicolumn{6}{|c|}{ Bone defect severity } \\
\cline { 2 - 7 } & \multicolumn{5}{|c|}{ Defect of the anterior rim of the glenoid less than 15 \% + Hill Sachs } \\
\cline { 2 - 7 } & $0-1$ grade & grade 2 & grade 3 & grade 0-1 & grade 2 & grade 3 \\
\hline Glenoid on track $(\mathrm{n}=137)$ & 48 & 70 & 17 & 9 & 1 & - \\
\hline Glenoid off track $(\mathrm{n}=32)$ & - & - & 2 & 9 & 5 \\
\hline
\end{tabular}
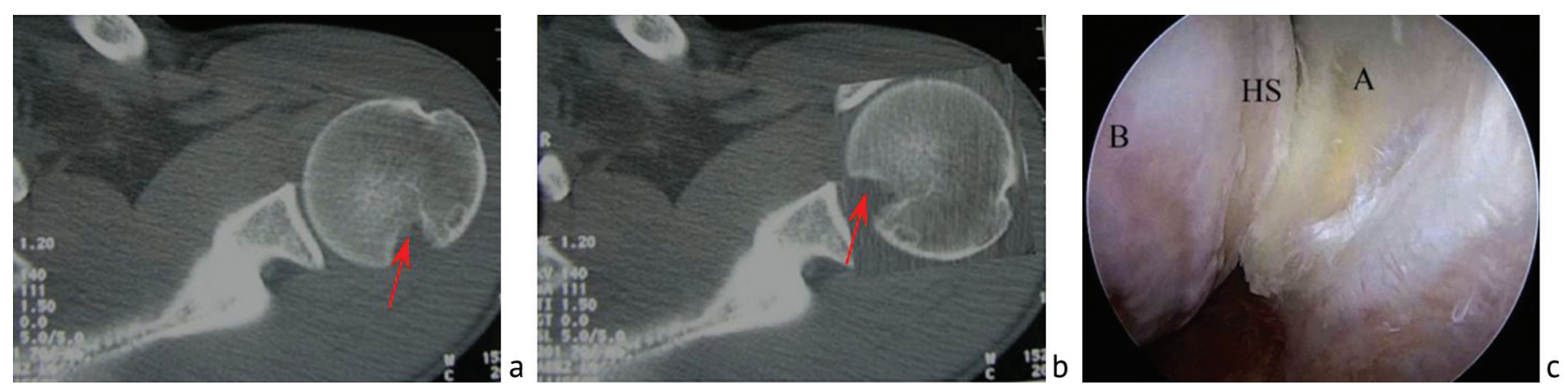

Fig. 6 Mechanism of shoulder dislocation upon contact of the Hill-Sachs defect with the anterior rim of the glenoid: $\boldsymbol{a}$ HillSachs defect (arrow) in neutral rotation of the shoulder; $\boldsymbol{b}$ position of the Hill-Sachs defect in hyperelasticity (external rotation of the shoulder $\left.90^{\circ}\right) ; c$ arthroscopic visualization of shoulder dislocation upon contact of the Hill-Sachs defect with the anterior rim of the glenoid (A - articular surface of the glenoid, B - head of the humerus, HS - Hill-Sachs defect) 
Algorithms of surgical techniques choice based on anatomic and functional changes in the shoulder joint

\begin{tabular}{|l|c|}
\hline \multicolumn{1}{|c|}{ Anatomic and functional disorders } & Types of interventions \\
\hline Defect of the anterior rim of the glenoid more than $15 \%$ & Latarjet \\
\hline Defect of the anterior rim of the glenoid less than $15 \%$ & Bankart \\
\hline Hill-Sachs defect of grade 3 & Remplissage \\
\hline Hyperelasticity & Remplissage \\
\hline
\end{tabular}

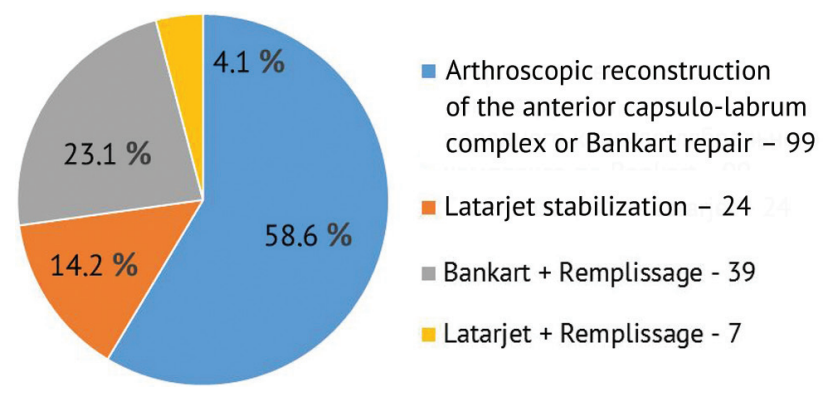

Fig. 7 Distribution of patients according to the types of interventions, $\mathrm{n}=169$

The results after interventions were studied in all the patients at a mean follow-up of $49.5 \pm 24.0$ months (range, 15-113 months). In the long term, eight patients complained of pain in the shoulder that was not persistent. Five patients felt pain after physical activities and three at weather change. Mean value of VAS score improved considerably. Also, there was an increase in the range of motion in the shoulder as compared with preoperative values. Mean range of external rotation decreased after the operation. Mean values of the evaluation scores improved as compared with the preoperative values (Table 5).

Table 5

Summary of long-term clinical results of surgical treatment of the recurrent anterior instability of the shoulder

\begin{tabular}{|l|c|}
\hline \multicolumn{1}{|c|}{ Parameter } & Value \\
\hline Mean flexion, $^{\circ}$ & $175.6 \pm 9.3$ \\
\hline Mean abduction, $^{\circ}$ & $173.0 \pm 12.3$ \\
\hline Mean external rotation, $^{\circ}$ & $55.9 \pm 14.4$ \\
\hline ROWE, points $^{\circ}$ & $89.9 \pm 17.5$ \\
\hline ASES, points & $94.0 \pm 7.1$ \\
\hline UCLA, points $^{\circ}$ & $32.7 \pm 3.7$ \\
\hline
\end{tabular}

Excellent and good outcomes at long term were achieved in $87.6 \%$ of patients. Stability of the involved shoulder joint in 132 patients (78.1\%) was comparable with the contralateral healthy one and they could continue sport and professional activities. Those patients expressed subjective satisfaction with the results. Twelve patients could not return to their amateur sports, among them 10 individuals were afraid of recurrence or motion restrictions, and two due to residual instability.

Seven patients $(4.1 \%)$ had poor results; three of them were associated with dislocation recurrence (after Latarjet - 1, after Bankart - 2). Residual subluxations in the joint along with restrictions in the functionality were the reasons of poor results in the other four patients (after Bankart - 3, after Bankart + Remplissage - 1) (Table 6).

Table 6

Distribution of patients according to ROWE in the long term after interventions

\begin{tabular}{|l|c|c|}
\hline $\begin{array}{c}\text { Postoperative result } \\
\text { (points) }\end{array}$ & $\begin{array}{c}\text { Number } \\
\text { of case }\end{array}$ & $\begin{array}{c}\% \text { from } \\
\text { the total }\end{array}$ \\
\hline Excellent (90-100) & 125 & 74.0 \\
\hline Good (75-89) & 23 & 13.6 \\
\hline Fair (51-74) & 14 & 8.3 \\
\hline Poor (0 50) & 7 & 4.1 \\
\hline
\end{tabular}

According to long-term follow-up radiography, 152 patients $(89.9 \%)$ did not have radiographic signs of shoulder osteoarthritis, 14 patients had mild osteoarthritis (stage 1) and three had moderate osteoarthritis (stage 2).

Lon-term CT-control was performed in 24 patients that underwent Latarjet procedure. Twenty (83.3\%) had consolidation of the bony junction and four patients $(1.7 \%)$ had fibrous union of the coracoid process graft. There was no graft osteolysis.

We detected only one complication associated with neuropathy of the axillary nerve and weakness of the deltoid muscle (after Bankart repair) that was treated by conservative neurotropic therapy for six months.

\section{DISCUSSION}

The most important conclusion of this study is the fact that the differentiated choice of the surgical technique based on anatomo-functional changes provides positive results of treatment in $95.9 \%$ of cases by minimal required surgical invasiveness. A ten-point ISIS for evaluation of instability severity was proposed for preoperative planning of surgical stabilization of the shoulder joint [14]. The designation of this system is a timely detection of patients that are at high risks of dislocation recurrence after arthroscopic Bankart repair. As reported, patients with ISIS score more than six points would have $70 \%$ of recurrence risks after Bankart operation. Therefore, such patients are recommended the Latarjet procedure. ISIS considers six risk factors, two of which are radiographic findings and four are data from anamnesis and clinical examination. Among 
significant radiographic signs are discontinuity of the contour of the inferior rim of the glenoid ( 2 points) and Hill-Sachs lesion seen in radiographs (2 points). According to our results of studying the radiographs of patients with chronic anterior instability of the shoulder, standard views did not show clearly the severity of bone defects. Thus, standard radiography revealed the defects of the anterior part of the glenoid only in 28 of our cases while CT/MRI showed it in 32 cases. On the contrary, CT/MRI revealed the Hill-Sachs defect of grade 3 in 24 patients while radiography showed it in 42 cases. It could be explained by the difficulty of interpretation of standard radiography findings. Jankauskas L. and co-authors conducted a radiographic evaluation of the defect of the anterior rim of the glenoid in 86 patients with anterior instability seen as a lost anterior sclerotic line of the articular process of the scapula and compared the findings with CT results. The study was done by two radiologists, one had assessed bone defects properly by radiographs in $56 \%$ of cases and the other in $64 \%$. The authors came to a conclusion that the discontinuity of the sclerotic line of the anterior part of the glenoid does not always correlate with bone tissue loss [22]. It is evident that imperfection in evaluation of bone defects in the shoulder due to restricted capabilities of radiography is a weak point of the ISIS system.

The findings of CT or MRI were used for assessment of bone defect severity in our study in order to develop an algorithm of surgical treatment. It allowed us to accurately define the impact of bone defects on the mechanisms of shoulder dislocation and to choose more properly the technique of stabilization for each case. If the ISIS system had been followed, we should have done 67 Latarjet procedures in our 169 patients. According to our developed algorithm to choose the intervention, Latarjet stabilization was performed in 31 patients. The Latarjet procedure is a more invasive intervention as compared with Bankart repair and is accompanied by forced disorders in the shoulder area anatomy [3,9]. Failures in reconstruction after a primary Latarjet operation may result in a future problematic scenario. And on the contrary, Latarjet operation may be easily performed in cases if arthroscopic stabilization fails. Therefore, one should be careful in choosing the technique and do not excessively use Latarjet as primary intervention.
In order to determine the anatomic relationship between the Hill-Sachs lesions and bone defect of the anterior glenoid, a concept of glenoid on track was proposed which is based on the grade of bone defects revealed by CT-study [23]. According to this theory, there is no risk of dislocation recurrence after soft-tissue stabilization if the Hill-Sachs lesion is within the glenoid track limits (the so called "glenoid on track"). If the Hill-Sachs lesion is out of the medial boundary of the glenoid track (glenoid off track), there is a high risk of recurrence after Bankart repair. Consequently, the technique to manage bone defects should be chosen in the latter case. Thus, James S. Shaha et al. studied twoyear results of Bankart arthroscopic reconstruction in 57 patients who had MRI for detection of bone defects and "glenoid track" condition before surgery. Recurrences occurred in 10 cases (18\%): in $6(75 \%)$ out of 8 patients with "glenoid off track" and only in $4(8 \%)$ patients out of 49 with "glenoid on track" [25].

The studied by us relationship of bone defects with "glenoid track" theory showed that the combination of the defect of the anterior rim of the glenoid more than $15 \%$ with Hill-Sachs lesion of grade 3, caused the "glenoid off track" impact in all such patients. In 30 out of 32 patents with "glenoid off track", there was the defect of the anterior rim of the glenoid more than $15 \%$. This finding allows us to consider a $15 \%$ anterior glenoid defect as critically important in the mechanism of dislocation, especially in combination with a grade 3 humeral head defect.

The undoubted advantage of the glenoid track theory is an accurate assessment of the effect of bipolar bone defects on instability in the shoulder joint. However, it does not take into account changes in the soft tissue component, first of all, the presence of hyperelasticity in the shoulder joint. In our study, during diagnostic arthroscopy in 9 patients with "glenoid on track" and hyperelasticity of the soft tissues of the shoulder joint, dislocations in the shoulder joint by the "off track" mechanism were observed. Therefore, we consider it compulsory to perform the remplissage procedure in case of hyperelasticity of the soft tissues of the shoulder joint, especially in combination with Hill-Sachs lesion.

\section{CONCLUSIONS}

Critically significant anatomical and functional disorders in patients with anterior instability of the shoulder joint are the defect of the anterior rim of the articular process of the scapula more than $15 \%$, the defect of the posterior surface of the humeral head (Hill-Sachs defect) of grade 3, hyperelasticity of the soft tissues of the shoulder joint. The severity of bone defects is directly proportional to the total number of dislocations. The effectiveness of surgical techniques to stabilize the shoulder joint, depending on the above anatomical and functional disorders, is $95.9 \%$. 


\section{REFERENCES}

1. Hatta T., Yamamoto N., Shinagawa K., Kawakami J., Itoi E. Surgical decision making based on the on-track/off-track concept for anterior shoulder instability: a case-control study. JSES Open Access, 2019, vol. 3, no. 1, pp. 25-28. DOI: 10.1016/j.jses.2018.10.001

2. Shaiakh A.B.S. MRT pri nestabilnosti plechevogo sustava [MRI for the shoulder instability]. Vestnik Rentgenologii i Radiologii, 2002, no. 6, pp. 3236. (in Russian)

3. Beliak E.A., Kubashev A.A., Lazko F.L., Lomtatidze E.Sh., Abdulkhabirov M.A., Ptitsyn K.A., Prizov A.P. Opyt primeneniia operatsii Latarzhe dlia lecheniia patsientov s perednei nestabilnostiu plechevogo sustava [The experience of performing Latarjet surgery for treating patients with anterior instability of the shoulder]. Travmatologiia i Ortopediia Rossii, 2014, no. 3, pp. 115-121. (in Russian)

4. Moore T.K., Hurley E.T., Rowe D.N., Hogan R.E., Kilkenny C.J., Pauzenberger L., Mullett H. Outcomes following arthroscopic Bankart repair in female patients. J. Shoulder Elbow Surg., 2020, vol. 29, no. 7, pp. 1332-1336. DOI: 10.1016/j.jse.2019.12.012

5. Ahmed I., Ashton F., Robinson C.M. Arthroscopic Bankart repair and capsular shift for recurrent anterior shoulder instability: functional outcomes and identification of risk factors for recurrence. J. Bone Joint Surg. Am., 2012, vol. 94, no. 14, pp. 1308-1315. DOI: 10.2106/JBJS.J.01983

6. DeFroda S., Bokshan S., Stern E., Sullivan K., Owens B.D. Arthroscopic Bankart Repair for the Management of Anterior Shoulder Instability: Indications and Outcomes. Curr. Rev. Musculoskelet. Med., 2017, vol. 10, no. 4, pp. 442-451. DOI: 10.1007/s12178-017-9435-2

7. Nakagawa S., Hirose T., Tachibana Y., Iuchi R., Mae T. Postoperative Recurrence of Instability due to New Anterior Glenoid Rim Fractures after Arthroscopic Bankart Repair. Am. J. Sports Med., 2017, vol. 45, no. 12, pp. 2840-2848. DOI: 10.1177/0363546517714476

8. Burkhart S.S., De Beer J.F. Traumatic glenohumeral bone defects and their relationship to failure of arthroscopic Bankart repairs: significance of the inverted-pear glenoid and the humeral engaging Hill-Sachs lesion. Arthroscopy, 2000, vol. 16, no. 7, pp. 677-694. DOI: 10.1053/jars.2000.17715

9. Rollick N.C., Ono Y., Kurji H.M., Nelson A.A., Boorman R.S., Thornton G.M., Lo I.K. Long-term outcomes of the Bankart and Latarjet repairs: a systematic review. Open Access J. Sports Med., 2017, vol. 8, pp. 97-105. DOI: 10.2147/OAJSM.S106983

10. Domos P., Ascione F., Wallace A.L. Arthroscopic Bankart repair with remplissage for non-engaging Hill-Sachs lesion in professional collision athletes. Shoulder Elbow, 2019, vol. 11, no. 1, pp. 17-25. DOI: 10.1177/1758573217728414

11. Barber F.A. Editorial Commentary: Open or Arthroscopic Surgery? Practice Makes Perfect... We Do Best What We Do Most Often. Arthroscopy, 2020, vol. 36, no. 3, pp. 872-874. DOI: 10.1016/j.arthro.2019.11.112

12. Wolf E.M., Arianjam A. Hill-Sachs remplissage, an arthroscopic solution for the engaging Hill-Sachs lesion: 2- to 10-year follow-up and incidence of recurrence. J. Shoulder Elbow Surg., 2014, vol. 23, no. 6, pp. 814-820. DOI: 10.1016/j.jse.2013.09.009

13. Pandey V., Gangadharaiah L., Madi S., Acharya K., Nayak S., Karegowda L.H., Willems W.J. A retrospective cohort analysis of arthroscopic Bankart repair with or without remplissage in patients with off-track Hill-Sachs lesion evaluated for functional outcomes, recurrent instability, and range of motion. J. Shoulder Elbow Surg., 2020, vol. 29, no. 2, pp. 273-281. DOI: 10.1016/j.jse.2019.06.005

14. Balg F., Boileau P. The instability severity index score. A simple pre-operative score to select patients for arthroscopic or open shoulder stabilization. J. Bone Joint Surg. Br., 2007, vol. 89, no. 11, pp. 1470-1477. DOI: 10.1302/0301-620X.89B11.18962

15. Ruiz Ibán M.A., Asenjo Gismero C.V., Moros Marco S., Ruiz Díaz R., Del Olmo Hernández T., Del Monte Bello G., García Navlet M., Ávila Lafuente J.L., Díaz Heredia J. Instability severity index score values below 7 do not predict recurrence after arthroscopic Bankart repair. Knee Surg. Sports Traumatol. Arthrosc., 2019, vol. 27, no. 12, pp. 3905-3911. DOI: 10.1007/s00167-019-05471-w

16. Oh J.H., Shin S.J., Cho C.H., Seo H.J., Park J.S., Rhee Y.G. Reliability of the Instability Severity Index Score as a Predictor of Recurrence after Arthroscopic Anterior Capsulolabral Reconstruction: A Multicenter Retrospective Study. Clin. Orthop. Surg., 2019, vol. 11, no. 4, pp. 445-452. DOI: $10.4055 /$ cios.2019.11.4.445

17. Itoi E. 'On-track' and 'off-track' shoulder lesions. EFORT Open Rev., 2017, vol. 2, no. 8, pp. 343-351. DOI: 10.1302/2058-5241.2.170007

18. Tikhilov R.M., Dokolin S.Iu., Kuznetsov I.A., Trachuk A.P., Burulev A.L., Mikhailova A.M., Zakharov K.I. Otdalennye rezultaty artroskopicheskogo lecheniia retsidiviruiushchei nestabilnosti plechevogo sustava, prichiny neudachnykh iskhodov [Long-term results of the arthroscopic treatment of the shoulder recurrent instability, reasons for unsuccessful outcomes]. Travmatologiia i Ortopediia Rossii, 2011, no. 1, pp. 5-13. (in Russian)

19. Calandra J.J., Baker C.L., Uribe J. The incidence of Hill-Sachs lesions in initial anterior shoulder dislocations. Arthroscopy, 1989, vol. 5, no. 4, pp. 254-257. DOI: 10.1016/0749-8063(89)90138-2

20. Momaya A.M., Tokish J.M. Applying the Glenoid Track Concept in the Management of Patients with Anterior Shoulder Instability. Curr. Rev. Musculoskelet. Med., 2017, vol. 10, no. 4, pp. 463-468. DOI: 10.1007/s12178-017-9436-1

21. Rowe C.R., Patel D., Southmayd W.W. The Bankart procedure: a long-term end-result study. J. Bone Joint Surg. Am., 1978 , vol. 60 , no. 1, pp. 1-16.

22. Richards R.R., An K.N., Bigliani L.U., Friedman R.J., Gartsman G.M., Gristina A.G., Iannotti J.P., Mow V.C., Sidles J.A., Zuckerman J.D. A standardized method for the assessment of shoulder function. J. Shoulder Elbow Surg., 1994, vol. 3, no. 6, pp. 347-352. DOI: 10.1016/S10582746(09)80019-0

23. Jankauskas L., Rüdiger H.A., Pfirrmann C.W., Jost B., Gerber C. Loss of the sclerotic line of the glenoid on anteroposterior radiographs of the shoulder: a diagnostic sign for an osseous defect of the anterior glenoid rim. J. Shoulder Elbow Surg., 2010, vol. 19, no. 1, pp. 151-156. DOI: $10.1016 /$ j.jse.2009.04.013

24. Yamamoto N., Itoi E., Abe H., Minagawa H., Seki N., Shimada Y., Okada K. Contact between the glenoid and the humeral head in abduction, external rotation, and horizontal extension: a new concept of glenoid track. J. Shoulder Elbow Surg., 2007, vol. 16, no. 5, pp. 649-656. DOI: 10.1016/j. jse.2006.12.012

25. Shaha J.S., Cook J.B., Rowles D.J., Bottoni C.R., Shaha S.H., Tokish J.M. Clinical Validation of the Glenoid Track Concept in Anterior Glenohumeral Instability. J. Bone Joint Surg. Am., 2016, vol. 98, no. 22, pp. 1918-1923. DOI: 10.2106/JBJS.15.01099

Received: 20.11 .2020

\section{Information about authors:}

1. Nikolay N. Chirkov, M.D., Ph.D.,

Federal Center for Traumatology, Orthopedics and Endoprosthetics, Cheboksary, Russian Federation, Chuvash State University named after I.N. Ulyanov, Cheboksary, Russian Federation, Email: surgenik@gmail.com

2. Nikolay S. Nikolaev, M.D., Ph.D., Professor,

Federal Center for Traumatology, Orthopedics and Endoprosthetics, Cheboksary, Russian Federation, Chuvash State University named after I.N. Ulyanov, Cheboksary, Russian Federation,

Email: nikolaevns@mail.ru

3. Alena V. Alekseeva, M.D.,

Federal Center for Traumatology, Orthopedics and Endoprosthetics, Cheboksary, Russian Federation, Email: alexeeva.shokolad@yandex.ru

4. Vladimir N. Yakovlev, M.D.,

Federal Center for Traumatology, Orthopedics and Endoprosthetics, Cheboksary, Russian Federation, Email: vnyakovlev@orthoscheb.com

5. Artur V. Lushin, M.D., Ph.D.,

Chuvash State University named after I.N. Ulyanov, Cheboksary, Russian Federation,

Email: lushin_cheb@mail.ru 